\title{
Interpersonal Predictors of Loneliness in Children : Variable- and Person-Centered Approaches
}

\author{
Fukuyama City University \\ Tatsuya Murakami \\ Kochi University of Technology \\ Shigeo Sakurai \\ University of Tsukuba
}

Takuma Nishimura ( $\square$ nishimu.takuma@gmail.com )

\section{Research Article}

Keywords: children, hierarchical linear modeling, loneliness, longitudinal study, mover-stayer latent transition analysis

Posted Date: September 1st, 2021

DOl: https://doi.org/10.21203/rs.3.rs-809687/v1

License: (9) This work is licensed under a Creative Commons Attribution 4.0 International License. Read Full License 


\section{Abstract}

The present study investigates potential interpersonal predictors of loneliness among late elementary school-aged children using variable-centered (hierarchical linear modeling; HLM) and person-centered (mover-stayer latent transition analysis) approaches. A total of 1,088 students (531 boys, 557 girls, $M_{\text {age }}$ $=10.35$, the age range was $9-11$ ) participated in a one-year survey. The results of the HLM revealed that victimization and relational aggression were positively correlated with loneliness, while positive peer relations were negatively correlated with loneliness. The mover-stayer analysis, however, showed that higher positive peer relations, lower victimization, and lower relational aggression do not necessarily relate to lower loneliness. Instead, the analysis only supported the findings in the inverse direction in which lower positive peer relations, higher victimization, and higher relational aggression are predictors of higher loneliness. We discuss a significance of a combination approach for sounding an alarm overdependence on the variable-centered approaches dominating child research and concluded the precautionary approach preventing the experience of loneliness, rather than reducing loneliness, is important for children in school education.

\section{Introduction}

Feelings of loneliness in children have been a major topic of interest in clinical and developmental psychology [11]. The reason is that the experience of loneliness is a sign of maladaptive functioning [5]; considerable research has revealed that loneliness is related to numerous problems of clinical relevance and risks including depression, anxiety, social withdrawal and suicide ideation [e.g., $4 ; 9 ; 13 ; 28]$. Additionally, lonely individuals tend to suffer from a cognitive bias toward threatening social stimuli, and easy access to the experience of loneliness [33]. According to van Dulmen and Goossens [36], $3-14 \%$ of children or adolescents were in a stable high or chronically high trajectory. Therefore, research involving its predictors is significant in terms of shedding light on how to prevent loneliness in children. The primary interest of the present paper was to find interpersonal predictors of loneliness in late elementary school-aged children through a combination of variable- and person- centered approaches.

\section{Predictors of Loneliness in Children}

Loneliness is a subjective experience and an emotional tendency described as sadness or pain, which is caused by an absence of connection with others [26]. As abundant research to date has focused on interpersonal experiences as predictors of loneliness, the primary trigger in children of loneliness is thought to be a lack of or difficulties in peer relations [1;2]. Under this assumption, which provides a rational for research regarding predictors of loneliness, positive peer relations (such as peer acceptance and social competence) and peer victimization are valid predictors of high loneliness. For example, Ladd, Kochenderfer, and Coleman [18] reported that the proportion of variance accounted by positive peer relations was of an above medium effect size in both cross-sectional and longitudinal studies and that victimization also predicted loneliness after half a year and changes in loneliness. Ladd and Troop- 
Gordon [19] conducted a five-year longitudinal study and found that chronic friendlessness and rejection accounted for a proportion of the variance in loneliness. Further, other studies using sociometric methods to obtain group members' ratings or nominations of liked or disliked others found that victimized children (i.e., those rejected) reported high loneliness compared to accepted or liked children [e.g., 6;25].

As a potential interpersonal predictor of loneliness, the predictive role of aggression remains unclear. Prinstein, Boergers, and Vernberg's [27] cross-sectional study showed that while victims of relational aggression are at risk of experiencing loneliness, there was no overall relation between aggression and Ioneliness. As recent research has suggested that aggression consists of two types [7]: overt aggression (e.g., punching, kicking, threatening) and relational aggression (e.g., excluding, manipulating others' relationships, spreading rumors), we focused on the relation between two types of aggression and loneliness.

In sum, as reviewed, loneliness in children is deeply related to interpersonal experience. Although many of other potential antecedents have been explored such as friendship quality, social network, and individual characteristics [8], the present study focused on the role of interpersonal predictors that have been a focus in considerable research as reviewed above: positive peer relations, victimization, and the two types of aggression (overt and relational aggression).

\section{Person-centered Approach}

While variable-centered approaches have dominated this research area, incorporation of person-centered approaches for interpretation has been recommended in the developmental psychopathology field [33]. The person-centered approaches focus on the identification of groups that share specific attributes or individual differences in developmental patterns [21]. As evident in Kochenderfer-Ladd and Wardrop's [15] study on victimization trajectories, one of the strengths of person-centered approaches was to provide more detailed insights into a relation. Besides adopting a variable-centered view, whereby they found a clear connection between victimization and loneliness, they took a person-centered view by focusing on trajectory patterns. This latter view illustrated that while children moving toward victimization status tended to experience greater loneliness, they did not necessarily become less lonely when moving away from victimization status.

In the first place, previous research on children's loneliness focusing on predictors seems to have relied on a variable-centered approach; furthermore, researchers have not given much attention to the potential over-interpretation of covariates. For example, when a negative longitudinal relation is found between an independent variable (i.e., potential predictors) and loneliness, researchers can interpret the relation as indicating both that a lower score on the independent variable is predictive of increased feelings of loneliness and that a higher score on the independent variable is predictive of decreased feelings of loneliness. This interpretation, however, is not always applicable because a mere correlation does not necessarily mean that both conditions are simultaneously satisfied; that is, only one condition is well established and then the negative relation emerges. Therefore, in addressing the issue of potential overinterpretation of covariates, person-centered views are useful. 
To investigate the predictors of loneliness from person-centered approach, mover-stayer latent trajectory analysis (mover-stayer LTA) is applicable. The mover-stayer LTA explores transitional patterns between latent profiles across time; it differs from typical LTA in being able to identify participants whose latent profiles change (i.e., movers) or remain the same (i.e., stayers) during the research period. Additionally, we can confirm whether the results of a variable-centered approach are in the same direction by applying mover-stayer LTA via comparison of the scores of potential predictors at specific time points. For example, if positive peer relations are strongly related to reduced loneliness (variable-centered view), individuals with latent profiles characterized by decreasing loneliness should have higher scores for positive peer relations during the period of the assessments (person-centered view).

The other advantage of mover-stayer LTA is that this method widely covers potential patterns of trajectories. Here, we reviewed seven empirical research that identified loneliness trajectories in children $[3 ; 10 ; 12 ; 17 ; 29 ; 32 ; 37]$, revealing specific subpopulation of trajectory patterns such as low levels of loneliness, decreasing, increasing, and chronically high patterns. All seven studies reported a trajectory of individuals with decreasing loneliness. However, sub-groups of loneliness trajectories were not consistent among the previous studies; for example, although five of them reported increasing trajectory, Harris et al. [10] and Ladd and Ettekal. [17] did not report the increasing pattern.

\section{Overview of the Present Study}

The purpose of the present study was to identify predictors of loneliness through variable- and personcentered approaches. First, using hierarchical linear modeling (HLM) as the variable-centered approach, we investigated the effect of positive peer relations, victimization, and the two types of aggression on loneliness. Second, using mover-stayer LTA as the person-centered approach, we tested the replication of the results by HLM. The hypothesis regarding predictors of loneliness in HLM was that victimization and relational aggression would be positively correlated, and positive peer relations would be negatively correlated with loneliness. In addition, we expected that the results of the mover-stayer LTA would support the direction of results of the HLM.

\section{Method}

\section{Study Design}

This study involved a one-year longitudinal design with three measurement points; the time interval between measurement points was about half a year. We conducted the baseline assessment (Time 0: T0) for loneliness between January and February, the second assessment (Time 1: T1) for loneliness and the potential predictors (i.e., positive peer relations, victimization, and two types of aggression) between June and July, and the third assessment (Time 2: T2) for loneliness a year after baseline between January and February. The reason why these interpersonal predictors were assessed at only $\mathrm{T} 1$ was to match the results of HLM and mover-stayer LTA.

\section{Participants}


A total of 1,088 elementary school students in the $4^{\text {th }}$ and $5^{\text {th }}$ grade $\left(531\right.$ boys and 557 girls; $M_{\text {age }}=10.35$, age range was 9 -11) from eight public schools (37 classes) in Japan participated in the survey. The number of participants with complete data was 1054 at T0, 1046 at T1, 1071 at T2, and 1029 throughout the three measurement points. All participants were Japanese and of lower to upper middle-class socioeconomic status. The sample was highly homogeneous in terms of ethnic and cultural back ground based on their demographics.

\section{Measures}

Loneliness (children's report). We used the Five-item Loneliness Scale for Children (Five-LSC; [24]). All five items use direct expressions to assess loneliness (e.g., "do you feel lonely?"). The scale has demonstrated sufficient validity based on teachers' observational assessment. According to Nishimura et al. [24], the difference in the score of loneliness between lonely students nominated by teachers and nonnominated was significant (Cohen's $d=0.60$ ). Also, loneliness assessed by the Five LSC was negatively correlated with social competence and social skills, and positively correlated with withdrawn behaviors. The rating was a 4-point Likert scale with ranging from 1 (strongly disagree) to 4 (strongly agree). We computed the arithmetic mean of the five items with higher scores reflecting higher loneliness.

Participants responded to the Five-LSC at all three time points. McDonald's omega which is one of the indicators of scale's internal consistency was .91 at T0, .93 at T1 and .94 at T2 in this study.

Positive peer relations (children's report). We assessed positive peer relations with a three-item scale (e.g., "Are peers kind to you") standardized and published by the Toshobunka Company in Japan. The scale has established clinical validity with low scores predicting peer victimization and failure to attend school. The rating is a 4-point with ranging from 1 (strongly disagree) to 4 (strongly agree). We computed the arithmetic mean of all three items with higher scores reflecting more positive peer relations. In this study, McDonald's omega of this scale was .83.

Victimization (children's report). We used a six-item scale of victimization standardized and published by the Tosyobunka Company in Japan. This scale has high clinical and prospective validity, with high scores predicting non-attendance and being bullied at school. The rating was a 4-point, ranging from 1 (strongly disagree) to 4 (strongly agree). We used arithmetic mean of all six items with higher scores reflecting higher victimization. In this study, McDonald's omega of this scale was .89 .

Relational and overt aggression (teachers' report). We used a teacher-report measure to assess children's relational and overt aggression in reference to Click and Grotpeter [7]. This measure consisted of seven items, four of which assessed relational aggression (e.g., tries to exclude others from their group), and three of which assessed overt aggression (e.g., hits, pushes others). Items are rated on a five-point scale, ranging from 1 (never) to 5 (always). We used the arithmetic mean with higher scores reflecting higher two types of aggression. Thirty-seven homeroom teachers responded to items for each student in their homeroom. In this study, McDonald's omega was .89 for relational aggression and .90 for overt aggression. 


\section{Procedure}

Homeroom teachers administered all questionnaire surveys in their class. The top sheet of the questionnaire contained the following statements: (a) the survey was not related to school grade evaluations, (b) the privacy of those taking the survey would be protected, and (c) participants were guaranteed the freedom to withdraw from participation. Participants provided demographics such as grade, class, sex, and student number on the last page of the questionnaire, which allowed us to link data from different time points and the teacher assessments.

This research project involved collaboration with the eight public schools. We contributed to each school's educational practice by providing feedback regarding homeroom management and identifying students with a high risk of school maladjustment using the data that we obtained. The schools explained this project to parents, who provided informed consent on behalf of their children and obtained agreement of participation of the study. Also, participants agreed to participate in the study after informing them that they have the full freedom to participate or withdraw at any time of their participation by a written consent. The institutional research board (IRB) at the authors' university approved the procedures. All methods were performed in accordance with the guidelines.

\section{Statistical Analysis}

All analyses were performed using R 3.1.0 and M-plus ver. 7.11. We used HLM to examine the predictors of loneliness, because children were nested within classrooms. In HLM, variance in individual-level outcomes is partitioned into child (within-level) and classroom (between-level) variance. Thus, we first calculated the intra-class coefficient (ICC) to assess the percentage of the between-level variance in loneliness explained. The result showed that variance at the between-level was .02; although this variance was small, we nevertheless applied HLM to avoid a type-1 error [16]. Furthermore, because of the nature of classrooms managed by homeroom teachers, we expected that between-level variances would moderate the connection between loneliness at T1 and T2. Thus, we were also interested in analyzing cross-level interactions. For ease of interpretation, between-level variables were centered at the grand mean in the analysis.

From person-centered approaches, we first run latent profile analysis (LPA) and then mover-stayer LTA. LPA examines the number of underlying subgroups of participants with similar patterns based on latent factor scores. For determining the number of profiles which had the best fit to the hypothesized model, we referred to Akaike's information criterion (AIC), Bayesian information criterion (BIC), adjusted BIC (aBIC), and the result of bootstrap likelihood ratio test (BLRT). Models with relatively lower AIC, BIC, and aBIC values fit better than models with higher values. The BLRT compares the improvement in fit between neighboring class models. The null hypothesis is that the $k$-profile solution fits better than a solution with $k-1$ profiles and it yields a $p$-value. Next, we used to mover-stayer LTA to examine the transition of participants between latent profiles at T0 and T2. A mover-stayer LTA uses a second-order latent class variables that classifies individuals as "movers" and "stayers". After that, we estimated the relations 
between the transition status identified by mover-stayer LTA and each score of the predictors: positive peer relations, victimization, relational and overt aggression at T1.

Finally, we addressed missing data by using the full information maximum likelihood method. We checked the pattern of missing data and comparison of loneliness score of T2 between children with nonmissing and missing at T0 and T1 and ascertained that the effect sizes of differences were small; therefore, we assumed that the data were missing at random.

\section{Results}

\section{Preliminary Analyses}

Table 1 presents the means, standard deviations, and correlations among all variables. We observed moderately positive correlations between loneliness each time point $(r=.44$ to $.62, p<.001)$. Positive peer relations were significantly negatively correlated with loneliness, while victimization was significantly positively correlated with loneliness. Neither type of aggression, except for relational aggression at T1, was correlated with loneliness.

\section{Predictors of Loneliness: Hierarchical Linear Modeling}

Table 2 presents the results of the HLM. After controlling for demographics and baseline variables, we found that loneliness at T1, victimization and relational aggression were positively correlated with loneliness at T2. Positive peer relations were negatively correlated with loneliness at T2. Overt aggression was not associated with loneliness at T2. Focusing on cross-level interactions, between-level victimization was positively correlated with the relation between loneliness at T1 and T2.

\section{Predictors of Loneliness: Mover-Stayer LTA}

As described above, we first investigated the number of profiles that best fit the data at T0 and T2. Although we first hypothesized that the number of profiles would be more than four, uninterpretable profiles emerged. Therefore, we compared the models from one- to three-profiles. Table 3 presents the results of the comparisons of the latent profiles. At both measurement points, AIC, BIC, and aBIC of the three-profile model were the smallest among models. We found a significant difference among the models via the BLRT. Accordingly, we performed the mover-stayer LTA using the three-profile model for exploring the transitions between these profiles across measurement points.

Table 4 presents the characteristics of each profile. Profile 1 was "low group" as characterized by low scores of loneliness (1.03 to 1.10); in total, 812 students at T0 and 792 students at T2 belonged to profile 1. Profile 2 was "relatively low group" as characterized by relatively low scores of loneliness (1.93 to 2.09); in total, 210 students at T0 and 215 students at T2 belonged to profile 2. Finally, profile 3 was "high group" as characterized by high scores of loneliness (2.95 to 3.35); in total, 66 students at T0 and 81 students at $\mathrm{T} 2$ belonged to profile 3 . Table 5 and Figure 1 present the trajectory patterns. Movers were those who transitioned from one profile to another across time. Stayers were those who remained in the 
same profile. Overall, 768 students (70.59\%) were classified as stayers (i.e., patterns 1, 5, and 9) and 219 students $(29.41 \%$ ) as movers including 175 increasers (patterns 2, 3, and 6) and 145 decreasers (patterns $4,7$, and 8$)$.

Next, we estimated factor scores of positive peer relations, victimization, relational and overt aggression in order to investigate the effects of children's interpersonal experiences at T1 on these trajectories of loneliness. Tables 6 presents the factor scores of positive peer relations and victimization, while Table 7 presents those for the two types of aggression for each profile along with the results of one sample $t$ tests and their effect size (i.e., Cohen's $d$ ). These factor scores represent distal means (from zero); $p$ value is associated with the null hypothesis, i.e., that the mean is equal to zero. Overall, we found several statistically significant effects for positive peer relations, victimization, and relational aggression, but not for overt aggression. The stayer in pattern 1 was associated with higher peer relations, lower victimization, and lower relational aggression. Meanwhile, other profiles of the stayer (i.e., patterns 5 and 9) were associated with lower positive peer relations and higher victimization but had no relation with either type of aggression. As for the increaser (i.e., patterns 2, 3, and 6), they were associated with lower positive peer relations, higher victimization (chance level in profile 2), and relational aggression. As for the decreaser (i.e., patterns 4,7 , and 8), those in patterns 7 and 8 were associated with higher victimization, while pattern 8 was also negatively correlated with positive peer relations. No significant effects were found for pattern 4. Surprisingly, despite the fact that they were decreaser, their factor scores of positive peer relations and victimization were not positive and negative, respectively.

\section{Discussion}

This study investigated the interpersonal predictors of loneliness in late elementary school-aged children via both variable- and person-centered approaches. In the following sections, we discuss the results of each analysis in turn, and then outline their advantages.

\section{Primary findings: Answers from HLM and Mover-stayer Analysis}

The results of HLM revealed that positive peer relations were negatively correlated with loneliness, while victimization and relational aggression were positively correlated with this dependent variable. Normally, these results would allow us to interpret that more positive peer relations as well as lower victimization and relational aggression reduce the risk of loneliness; however, care must be taken in interpreting these relations, because the results of the mover-stayer LTA did not fully uphold the interpretation. As shown in Tables 7 and 8, the factor scores of positive peer relations of decreaser (i.e. patterns 4, 7, and 8) were not significantly positive (rather negative) while the scores of victimization and relational aggression of decreaser were not significantly negative. If higher positive peer relations contribute to lower loneliness, their factor scores should be positive; likewise, if lower victimization and relational aggression contribute to lower loneliness, their factor scores should be negative. Instead, the analysis supported only the inverse direction; that is, lower positive peer relations, and higher victimization and relational aggression were associated with higher loneliness. As shown, the factor scores of positive peer relations of increaser 
(i.e. patterns 2, 3, and 6) were significantly negative; similarly, the factor scores of victimization and relational aggression of increaser were significantly positive except for pattern 2 for victimization (but positive with small effect size; Cohen's $d=0.16$ ). Summarizing these findings, both approaches appear to support only the predictors of increased loneliness, but not those of decreased. As suggested by Kochenderfer-Ladd and Wardrop [15], these results caution us to over-interpretation by variable-centered approaches and indicate that a combination approach is a more useful and prudent approach on the data.

Based on the findings found in the result of within-level in HLM and mover-stayer LTA, the relation between victimization and loneliness at the cross-level interaction possibly indicates that a homeroom class characterized by higher victimization strengthens the relation in loneliness between T1 and T2. Generally, children with negative peer experiences tend to have distorted social cognitions [20] and are receptive to negative peer feedback [14] and social threat [30]. Classrooms with a higher score of victimization may evince continually rough or disrupted, in turn might make children with higher loneliness become more sensitive and over-evaluate their lonely experience. Taken together, these results indicate an importance of preventive practices using not only interpersonal intervention but also coordination of class room environment.

\section{Limitations}

The present study had the following limitations. First, we used subjective assessments of loneliness, positive peer relations, and victimization which likely led to inflated covariance. Additionally, although we used teachers' assessments for the two types of aggression, teacher report is not the only prototype for such assessment; future studies should test robustness of the findings by multiple assessments (e.g., observations, and teachers', parents', and peers' reports). Notably, the present research did not identify a predictor of reduced loneliness supported by both variable- and person-centered approaches. Specifically, some children shifted from higher to lower loneliness despite experiencing victimization and less positive peer relations. Future research should strive to identify the interpersonal predictors that explain this phenomenon to provide practical implications.

\section{Conclusion}

From variable-centered approach, this study identified the following variables as interpersonal predictors of decreasing of future loneliness: more positive peer relations, lower victimization, and lower relational aggression; however, by taking person-centered approach, we did not support the finding. Conversely, the person-centered approach indicated findings in the inverse direction in which less positive peer relations, higher victimization, and higher relational aggression are predictors of higher future loneliness. In other words, even if we find negative relations between potential predictors and loneliness in future studies, we should not interpret them in the desired direction without a deep consideration. The present study shows that using a combination of variable- and person-centered approaches provides insightful contributions and sounds an alarm over-dependence on the variable-centered approach. Moreover, we did not reveal 
decisive interpersonal indicators reducing loneliness. This means that, before experiencing loneliness, precautionary practices are important in educational practices.

\section{Declarations}

\section{Availability of the data and materials}

The datasets used and/or analyzed during the current study are available anytime from the corresponding author on reasonable request.

\section{Ethics approval and consent to participate}

This study has been approved from the Scientific Research Committee at University of Tsukuba. The subject number is 23-146. Participants agreed to participate in the study after informing them that they have the full freedom to participate or withdraw at any time of their participation.

\section{Conflicts of Interests}

The authors declare that they have no conflicts of interest.

The data that support the findings of this study are available on request.

\section{Acknowledgements}

This research was supported by a grant from Center of Developmental Education and Research (CODER) in Japan.

\section{Consent for publication}

All participants acknowledged their agreement to publish the results of the study after they were informed in writing and verbally that the study will be published in one of the refereed journals

\section{Authors' contributions}

The first author was involved in collecting data, analysis and preparing the first manuscript.

The second author performed the statistical analyses and helped in drafting the manuscript.

The third author participated in the design and coordination of the study and helped in drafting the manuscript.

\section{References}

1. Asher, S. R., \& Paquette, J. A. (2003). Loneliness and peer relations in Childhood. Current Directions in Psychological Science. 12(3), 75-78. 
2. Asher, S. R., Parkhurst, R., Hymel, J. T., \& Williams, G. A. (1990). Peer rejection and loneliness in childhood. In S. R. Asher \& J. D. Coie (Eds.), Peer rejection in childhood (pp. 253-274). New York: Cambridge University Press.

3. Benner, A. D. (2011). Latino adolescents' loneliness, academic performance, and the buffering nature of friendships. Journal of Youth Adolescence, 40(5), 556-567.

4. Cacioppo, J.T., Cacioppo, S., Cole, S.W., Capitanio, J.P., Coossens, L., \& Boomsma, D. I. (2015). Loneliness across phylogeny and a call for comparative studies and animal models. Perspectives on Psychological Science, 10(2), 202-212.

5. Cacioppo, S., Cacioppo, A. J., London, S., Goossens, L., \& Cacioppo, J. T. (2015). Loneliness: clinical import and interventions. Psychological Science, 10(2), 238-249.

6. Crick, N. R., \& Ladd, G. W. (1993). Children's perceptions of their peer experiences: Attributions, loneliness, social anxiety, and social avoidance. Developmental Psychology, 29(2), 244-254.

7. Crick, N. R., \& Grotpeter, J. K. (1995). Relational aggression, gender, and social-psychological adjustment. Child Development, 66(3), 710-722.

8. de Jong Gierveld, J, van Tilburg, T.G, \& Dykstra, P.A. (2018). Loneliness and social isolation: New ways of theorizing and conducting research. In Cambridge handbook of personal relationships, 2nd Ed (pp. 391-404).

9. Gallagher, M., Prinstein, M. J., Simon, V., \& Spirito, A. (2014). Social anxiety symptoms and suicidal ideation in a clinical sample of early adolescents: examining loneliness and social support as longitudinal mediators. Journal of Abnormal Child Psychology, 42(6), 871-83.

10. Harris, R. A., Qualter, P., Robinson, S. J. (2013). Loneliness trajectories from middle childhood to preadolescence: impact on perceived health and sleep disturbance. Journal of Adolescence, 36(6), 1295-1304.

11. Heinrich, L. M., \& Gullone, E. (2006). The clinical significance of loneliness: A literature review. Clinical Psychology Review, 26(6), 695-718.

12. Jobe-Shields, L., Cohen, R., \& Parra, G. (2011). Patterns of Change in Children's Loneliness: Trajectories from Third through Fifth Grades. Merrill Palmer Quarterly, 57(1), 25-47.

13. Jones, A. C., Schnka, K. C., van Dulmen, M. H., Bossarte, R. M., \& Swahn, M. H. (2011). Changes in loneliness during middle childhood predict risk for adolescent suicidality indirectly through mental health problems. Journal of Clinical Child \& Adolescent Psychology, 4O(6), 818-824.

14. Juvonen, J. \& Galván, A. (2009). Bullying as a means to foster compliance. In M. J. Harris (Ed.), Bulling, rejection, and peer victimization: A social cognitive neuroscience perspective (pp. 299-318). New York: Springer Publishing Company.

15. Kochenderfer-Ladd, B., \& Wardrop, J. L. (2001). Chronicity and instability of children's peer victimization experiences as predictors of loneliness and social satisfaction trajectories. Child Development, 72(1), 134-151.

16. Kreft, I., \& de Leeuw, J. (1998). Introducing multilevel modeling. London: Sage 
17. Ladd, G. W., Ettekal, I. (2013). Peer-related loneliness across early to late adolescence: normative trends, intra-individual trajectories, and links with depressive symptoms. Journal of Adolescence, 36(6), 1269-1282.

18. Ladd, G. W., Kochenderfer-Ladd, B., \& Coleman, C. C. (1997). Classroom peer acceptance, friendship, and victimization: Distinct relational systems that contribute uniquely to children's school adjustment? Child Development, 68(6), 1181-1197.

19. Ladd, G. W., \& Troop-Gordon, W. (2003). The role of chronic peer difficulties in the development of children's psychological adjustment problems. Child Development, 74(5), 1344-1367.

20. Lansu, T. A., van Noorden, T. H., \& Deutz, M. H. (2017). How children's victimization relates to distorted versus sensitive social cognition: Perception, mood, and need fulfillment in response to Cyberball inclusion and exclusion. Journal of Experimental Child Psychology, 154, 131-145.

21. Laursen, B. P., \& Hoff, E. (2006). Person-centered and variable-centered approaches to longitudinal data. Merrill-Palmer Quarterly, 52(3), 377-389.

22. Mahon, N. E., Yarcheski, A., Yarcheski, T. J., Cannella, B. L., \& Hanks, M. M. (2006). A meta-analytic study of predictors for loneliness during adolescence. Nursing Research, 55(5), 308-315.

23. Nangle, D. W., Erdley, C. A., Newman, J. E., Mason, C. A., \& Carpenter, E. M. (2003). Popularity, friendship quantity, and friendship quality: Interactive influences on children's loneliness and depression. Journal of Clinical Child and Adolescent Psychology, 32(4), 546-555.

24. Nishimura, T., Murakami, T., \& Sakurai, S. (2015). The development of the Five-item Loneliness Scale for Children. Japanese Journal of Psychology, 86(4), 368-373.

25. Parkhurst, J. T., \& Asher, S. R. (1992). Peer rejection in middle school: Subgroup differences in behavior, loneliness, and interpersonal concerns. Developmental Psychology, 28(2), 231-241.

26. Parkhurst, J. T., \& Hopmeyer, A. (1999). Developmental change in the sources of loneliness in childhood and adolescence: Constructing a theoretical model. In Rotenberg, K. J., Hymel, S. (Eds.), Loneliness in childhood and adolescence (pp. 56-79). New York: Cambridge, University Press.

27. Prinstein, M. J., Boergers, J. \& Vernberg, E. M. (2001). Overt and relational aggression in Adolescents: Social-psychological adjustment of aggressors and victims. Journal of Clinical Child Psychology, 30(4), 479-491.

28. Qualter, P., Brown, S. L., Munn, P., \& Rotenberg, K. J. (2010). Childhood loneliness as a predictor of adolescent depressive symptoms: an 8-year longitudinal study. European Child \& Adolescent Psychiatry, 19(6), 493-501.

29. Qualter, P., Brown, S. L., Rotenberg, K. J., Vanhalst, J., Harris, R. A., Goossens, L., Bangee, M., \& Munn, P. (2013). Trajectories of loneliness during childhood and adolescence: Predictors and health outcomes. Journal of Adolescence, 36(6), 1283-1293.

30. Qualter, P., Rotenberg, K., Barrett, L., Henzi, P., Barlow, A., Stylianou, M., \& Harris, R. A. (2013). Investigating hypervigilance for social threat of lonely children. Journal of Abnormal Child Psychology. 41(2), 325-338. 
31. Rose, A. J., Swenson, L. P., \& Waller, E. M. (2004). Overt and relational aggression and perceived popularity: developmental differences in concurrent and prospective relations. Developmental Psychology, 40(3), 378-387.

32. Schinka, K. C., van Dulmen, M. H., Mata, A. D., Bossarte, R., \& Swahn, M. (2013). Psychosocial predictors and outcomes of loneliness trajectories from childhood to early adolescence. Journal of Adolescence, 36(6), 1251-1260.

33. Spithoven, A. W. M., Bijttebier, P., \& Goossens L. (2017). It is all in their mind: A review on information processing bias in lonely individuals. Clinical Psychology Review, 58, 97-114.

34. Sterba, S. K., \& Bauer, D. J. (2010). Matching method with theory in person-oriented developmental psychopathology research. Development and Psychopathology, 22(2), 239-254.

35. Terrell-Deutsch, B. (1999). The conceptualization and measurement of childhood loneliness. In Rotenberg, K. J., Hymel, S. (Eds.), Loneliness in childhood and adolescence (pp. 11-33). New York: Cambridge University Press.

36. Van Dulmen, M. H., \& Goossens, L. (2013). Loneliness trajectories. Journal of Adolescence, 36(6), 1247-1249.

37. Vanhalst J., Goossens, L., Luyckx, K., Scholte, R. H. J., \& Engels, R. C. M. E. (2013). The development of loneliness from mid- to late adolescence: trajectory classes, personality traits, and psychosocial functioning. Journal of Adolescence, 36(6), 1305-1312.

\section{Tables}

Table 1

Means, Standard Deviations, $95 \%$ Confidence Intervals and Correlations A mong the V ariables

\begin{tabular}{|c|c|c|c|c|c|c|c|c|c|}
\hline & $M$ & $S D$ & $95 \% \mathrm{CI}$ & 2 & 3 & 4 & 5 & 6 & 7 \\
\hline 1. Loneliness ( $\mathrm{T} 0$ : baseline) & 1.38 & 0.62 & {$[1.34,1.42]$} & $.55^{* * *}$ & $-.36^{* * *}$ & $.43^{* * *}$ & .02 & .01 & $.44^{* * *}$ \\
\hline 2. Loneliness (T1) & 1.36 & 0.60 & {$[1.32,1.39$ ] } & & $-.50^{* * *}$ & $.65^{* * *}$ & .06 & .04 & $.62^{* * *}$ \\
\hline 3. Positive peer relations (T1) & 3.33 & 0.57 & {$[3.30,3.37$ ] } & & & $-.49 * * *$ & -.04 & -.06 & $-.42^{* * *}$ \\
\hline 4. Victimization (T1) & 1.51 & 0.57 & {$[1.47,1.54]$} & & & & .04 & .06 & $.50^{* * *}$ \\
\hline 5. Relational aggression (T1) & 1.40 & 0.59 & {$[1.37,1.44]$} & & & & & $.47 * * *$ & $.12^{* * *}$ \\
\hline 6. Overt aggression (T1) & 1.15 & 0.40 & {$[1.13,1.18]$} & & & & & & .06 \\
\hline 7. Loneliness (T2) & 1.41 & 0.66 & {$[1.37,1.45]$} & & & & & & \\
\hline
\end{tabular}

Note. $\mathrm{T} 0=\mathrm{Time} 0, \mathrm{~T} 1=\mathrm{Time} 1, \mathrm{~T} 2=$ Time $2 . \mathrm{CI}=$ confidence interval

$* * * p<.001, * * p<.01, * p<.05$ 
Table 2

The Results of HierarchicalLiner Modeling for Loneliness at Time 2

\begin{tabular}{rllll}
\hline variables & $\gamma$ & $S E$ & $p$ & $95 \% \mathrm{CI}$ \\
\hline
\end{tabular}

Within level

(Demographics \& baseline variables)

Gender

Age

loneliness ( $\mathrm{T} 0$ : baseline)

(T1 variables)

Loneliness

Positive peer relations

Victimization

Relational aggression

Overt aggression

Cross-level interaction

Positive peer relations with loneliness (T1)

Victimization with loneliness (T1)

Relational aggression with loneliness (T1)

Overt aggression with loneliness (T1)

\begin{tabular}{|c|c|c|c|c|}
\hline-0.08 & 0.03 & .010 & {$[-0.14$} & $-0.02]$ \\
\hline-0.01 & 0.03 & .749 & {$[-0.06$,} & $0.05]$ \\
\hline 0.11 & 0.03 & $<.001$ & 0.05 & $0.17]$ \\
\hline
\end{tabular}

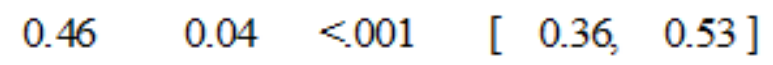

$-0.13 \quad 0.03<001 \quad\left[\begin{array}{lll}-0.20, & -0.07]\end{array}\right.$

$\begin{array}{llll}0.11 & 0.04 & .002\end{array}\left[\begin{array}{ll}0.04, & 0.19\end{array}\right]$

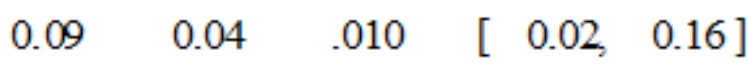

$\begin{array}{llll}0.03 & 0.05 & .719 & {\left[\begin{array}{ll}-0.06, & 0.13\end{array}\right]}\end{array}$

\begin{tabular}{|c|c|c|c|c|}
\hline 0.42 & 0.32 & .192 & [ - 0.21, & $1.04]$ \\
\hline 0.82 & 0.35 & .020 & [ 0.13 , & $1.50]$ \\
\hline-0.09 & 0.08 & .915 & {$[-0.42$,} & $0.25]$ \\
\hline-0.11 & 0.33 & .740 & {$[-0.74$} & 0.53 \\
\hline
\end{tabular}

Note. $\mathrm{T} 0=\mathrm{T}$ ime $0, \mathrm{~T} 1=\mathrm{T}$ ime $1 . \mathrm{CI}=$ confidence interval 
Table 3

The Summaries of Model Comparison of Latent Profile Models at both T0 and T2

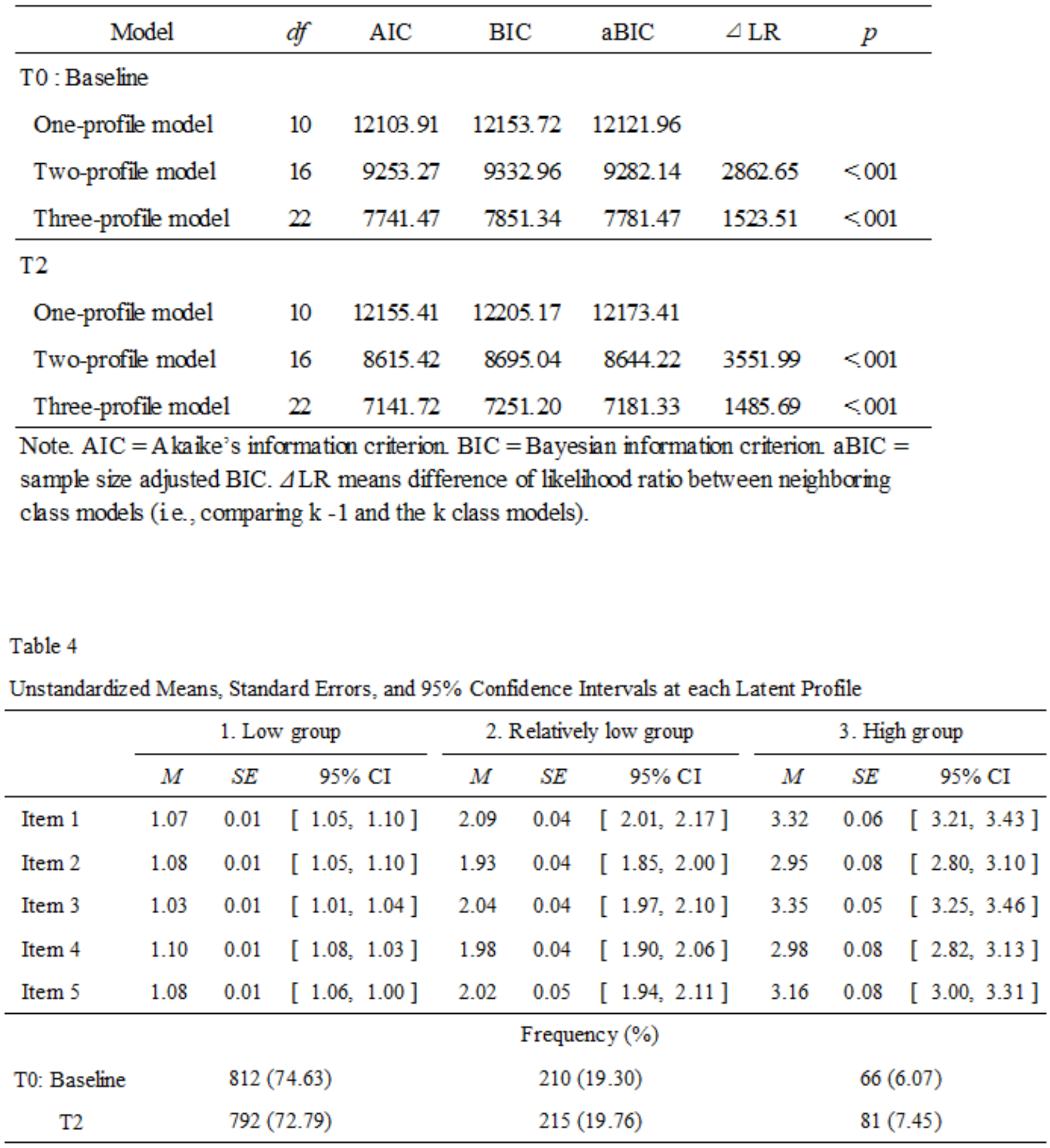

Note. $\mathrm{T} 0=\mathrm{T}$ ime $0, \mathrm{~T} 2=\mathrm{Time} 2 . \mathrm{CI}=$ confidence interval 


\section{Table 5}

Frequency of Each Mover-Stayer Latent Transition Pattern

\begin{tabular}{|c|c|c|c|c|c|}
\hline \multicolumn{3}{|c|}{ Latent profile } & \multirow{2}{*}{ Frequecny } & \multirow{2}{*}{$\%$} & \multirow{2}{*}{$\begin{array}{c}\text { Change of } \\
\text { category }\end{array}$} \\
\hline No. & T0: Baseline & T2 & & & \\
\hline 1 & Low group & Low group & $671^{2}$ & 61.67 & Stayer \\
\hline 2 & & Relateively low group & $117^{\mathrm{b}}$ & 10.75 & Increaser \\
\hline 3 & & High group & $24^{\mathrm{b}}$ & 2.21 & Increaser \\
\hline 4 & Relateively low group & Low group & $102^{\mathrm{b}}$ & 9.38 & Decreaser \\
\hline 5 & & Relateively low group & $74^{2}$ & 6.80 & Stayer \\
\hline 6 & & High group & $34^{b}$ & 3.13 & Increaser \\
\hline 7 & High group & Low group & $19^{b}$ & 1.75 & Decreaser \\
\hline 8 & & Relateively low group & $24^{b}$ & 2.21 & Decreaser \\
\hline 9 & & High group & $23^{2}$ & 2.11 & Stayer \\
\hline
\end{tabular}

Note. ${ }^{\text {a }}$ denotes "stayers" : those participants who were classified into the same latent profile in T0 (baseline) and T2. ${ }^{b}$ denotes "movers" : those participants who were classified into the different latent profile from $\mathrm{T} 0$ (baseline) and $\mathrm{T} 2$.

Table 6

Results of One Sample t-test for Factor Scores of Positive Peer Relations and Victimization at Each Profile

\begin{tabular}{|c|c|c|c|c|c|c|c|c|c|c|c|c|c|}
\hline \multicolumn{2}{|c|}{ Profiles } & \multicolumn{6}{|c|}{ Positive peer relations } & \multicolumn{6}{|c|}{ Victimization } \\
\hline No. & Patterns & $M$ & $S D$ & $95 \% \mathrm{CI}$ & $t$ & $p$ & $d$ & $M$ & $S D$ & $95 \% \mathrm{CI}$ & $t$ & $p$ & $d$ \\
\hline 1 & $L-L$ & 0.18 & 0.39 & {$[0.15,0.21]$} & 11.63 & $<.001$ & 0.45 & -0.21 & 0.38 & {$[-0.24,-0.18]$} & -14.16 & $<.001$ & 0.55 \\
\hline 2 & $L-R L$ & -0.10 & 0.45 & {$[-0.19,-0.02]$} & -2.44 & .016 & 0.23 & 0.07 & 0.44 & {$[-0.01,0.15]$} & 1.72 & .088 & 0.16 \\
\hline 3 & $\mathrm{~L}-\mathrm{H}$ & -0.62 & 0.84 & {$[-0.98,-0.26]$} & -3.60 & .002 & 0.73 & 0.73 & 0.86 & {$[0.37,1.01]$} & 4.18 & $<.001$ & 0.85 \\
\hline 4 & RL - L & -0.04 & 0.52 & {$[-0.15,0.06]$} & -0.87 & .387 & 0.09 & 0.01 & 0.49 & {$[-0.09,0.10]$} & 0.18 & .857 & 0.02 \\
\hline 5 & $R L$ - RL & -0.40 & 0.48 & {$[-0.51,-0.29]$} & -7.20 & $<.001$ & 0.84 & 0.48 & 0.54 & {$[0.35,0.60]$} & 7.62 & $<.001$ & 0.89 \\
\hline 6 & $\mathrm{RL}-\mathrm{H}$ & -0.60 & 0.60 & {$[-0.81,-0.39]$} & -5.81 & $<.001$ & 1.00 & 0.70 & 0.57 & {$[0.50,0.90]$} & 7.11 & $<.001$ & 1.22 \\
\hline 7 & $\mathrm{H}-\mathrm{L}$ & -0.16 & 0.55 & {$[-0.42,0.10]$} & -1.29 & .214 & 0.30 & 0.31 & 0.50 & {$[0.06,0.55]$} & 2.64 & .018 & 0.61 \\
\hline 8 & $H-R L$ & -0.62 & 0.56 & {$[-0.86,-0.38]$} & -5.41 & $<.001$ & 1.10 & 0.94 & 0.70 & {$[0.64,1.24$ ] } & 6.51 & $<.001$ & 1.33 \\
\hline 9 & $\mathrm{H}-\mathrm{H}$ & -0.84 & 0.66 & {$[-1.12,-0.55]$} & -6.06 & $<.001$ & 1.26 & 1.16 & 0.69 & {$[0.86,1.46]$} & 8.29 & $<.001$ & 1.73 \\
\hline
\end{tabular}

Note. $\mathrm{L}=\mathrm{L}$ ow group, $\mathrm{RL}=$ Relatively low group, $\mathrm{H}=$ High group. $\mathrm{CI}=$ confidence interval 
Table 7

Results of One Sample t-test for Factor Scores of Two Types of Aggression at Each Profile

\begin{tabular}{|c|c|c|c|c|c|c|c|c|c|c|c|c|c|}
\hline \multirow{2}{*}{\multicolumn{2}{|c|}{$\frac{\text { Profiles }}{\text { No. patterns }}$}} & \multicolumn{6}{|c|}{ Relational aggression } & \multicolumn{6}{|c|}{ Overt aggression } \\
\hline & & $M$ & $S D$ & $95 \% \mathrm{CI}$ & $t$ & $p$ & $d$ & $M$ & $S D$ & $95 \% \mathrm{CI}$ & $t$ & $p$ & $d$ \\
\hline 1 & $\mathrm{~L}-\mathrm{L}$ & -0.06 & 0.57 & {$[-0.10,-0.02]$} & -2.73 & .007 & 0.11 & -0.04 & 0.62 & {$[-0.08,0.01]$} & -1.74 & .081 & 0.07 \\
\hline 2 & $\mathrm{~L}-\mathrm{RL}$ & 0.19 & 0.67 & {$[0.07,0.31]$} & 3.06 & .003 & 0.28 & 0.14 & 0.99 & {$[-0.04,0.32]$} & 1.50 & .136 & 0.14 \\
\hline 3 & $\mathrm{~L}-\mathrm{H}$ & 0.61 & 0.79 & {$[0.28,0.95$ ] } & 3.83 & $<.001$ & 0.78 & 0.43 & 1.24 & {$[-0.10,0.95]$} & 1.68 & .101 & 0.34 \\
\hline 4 & $R L-L$ & -0.04 & 0.59 & {$[-0.16,0.07]$} & -0.77 & .442 & 0.08 & 0.03 & 0.73 & {$[-0.11,0.17]$} & 0.42 & .673 & 0.04 \\
\hline 5 & $R L$ - RL & -0.09 & 0.66 & {$[-0.24,0.06]$} & -1.16 & .250 & 0.13 & -0.12 & 0.59 & {$[-0.26,0.02]$} & -1.74 & .085 & 0.20 \\
\hline 6 & $\mathrm{RL}-\mathrm{H}$ & 0.44 & 0.72 & {$[0.19,0.69]$} & 3.53 & .001 & 0.61 & 0.27 & 1.06 & {$[-0.10,0.65]$} & 1.50 & .143 & 0.26 \\
\hline 7 & $\mathrm{H}-\mathrm{L}$ & 0.10 & 0.75 & {$[-0.26,0.46]$} & 0.58 & .571 & 0.13 & -0.08 & 0.40 & {$[-0.27,0.11]$} & -0.87 & .395 & 0.20 \\
\hline 8 & $H-R L$ & -0.05 & 0.41 & {$[-0.22,0.13]$} & -0.57 & .574 & 0.12 & -0.04 & 0.45 & {$[-0.23,0.15]$} & -0.42 & .680 & 0.09 \\
\hline 9 & $\mathrm{H}-\mathrm{H}$ & -0.05 & 0.54 & {$[-0.28,0.18]$} & -0.45 & .656 & 0.09 & 0.04 & 0.72 & {$[-0.27,0.35]$} & 0.26 & .795 & 0.05 \\
\hline
\end{tabular}

Note. $\mathrm{L}=\mathrm{L}$ ow group, $\mathrm{RL}=$ Relatively low group, $\mathrm{H}=$ High group. $\mathrm{CI}=$ confidence interval

Figures

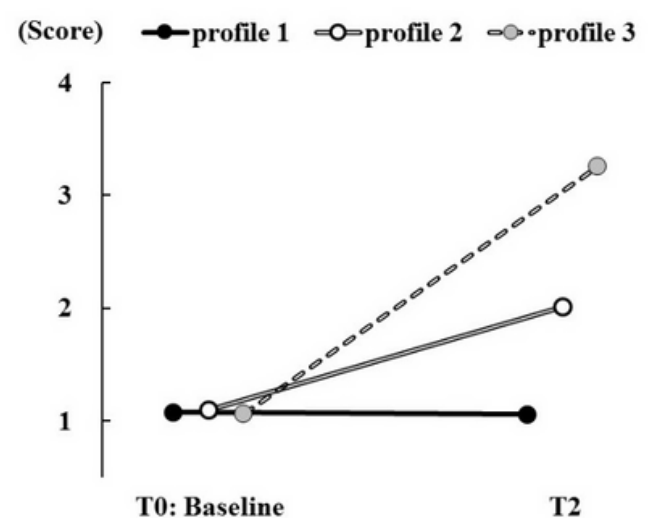

T0: Baseline

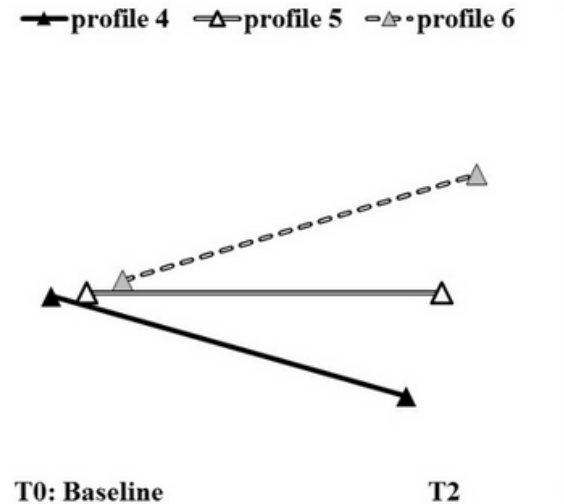

T0: Baseline $\rightarrow$ profile $7 \Leftrightarrow$ profile $8 \Leftrightarrow$ oprofile 9

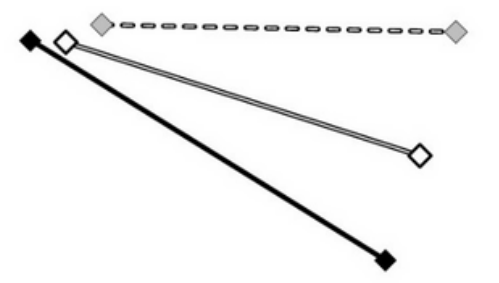

T0: Baseline

Figure 1

All transition patterns of mover-stayer LTA 